\title{
ACTITUD Y PRÁCTICA SOLIDARIA EN LOSIAS JÓVENES ANDALUCES
}

\section{SOLIDARITY ATTITUDE AND PRACTICES IN ANDALUSIAN YOUTH}

\author{
José Manuel Corpas Nogales \\ Universidad de Granada \\ joscorpas@ugr.es
}

\begin{abstract}
Resumen: Esta investigación versa sobre la construcción social de la juventud andaluza en torno a las prácticas solidarias desde una perspectiva integradora de actitud y práctica. Por ello el objetivo principal de este trabajo se centra en describir la actitud y las prácticas solidarias de la población juvenil considerando a la juventud como una construcción social. Para conseguir este objetivo hemos desarrollado una metodología cualitativa utilizando entrevistas personales y grupos de discusión. Además, hemos llevado a cabo una triangulación metodológica realizando un análisis comparativo de los datos obtenidos con los datos aportados por las fuentes secundarias. Como conclusión principal resaltamos la influencia que presenta la construcción negativa de la juventud en el momento de pensar y de realizar prácticas solidarias.
\end{abstract}

Palabras clave: Juventud, solidaridad, constructivismo, voluntariado, ONG,s. Andalucía.
Abstract: This research deals with the social construction of the Andalusian youth concerning the solidarity in practice from an integrative perspective of both attitude and practice. Thus the main aim of this research is focused on the description of the solidarity attitude and solidarity in practice of youngsters, who are considered to be a social construction. In order to achieve this aim, a qualitative methodology using interviews and discussion groups has been carried out. Furthermore a comparative analysis between the data obtained and the data from secondary sources was done via a methodological triangulation. As the main conclusion we stand out the influence that negative construction presents of young people in the moment of thinking and making solidary practices.

Key words: youth, solidarity, constructivism, voluntary service, NGOs, Andalusian. 


\section{Introducción}

Esta investigación trata sobre los/as jóvenes andaluces y la vinculación de éstos con la solidaridad. Concretamente hemos realizado un análisis basado en una perspectiva integradora de actitud y práctica a través de una visión constructivista, mediante la cual visualizamos la juventud como un constructo social. Por lo tanto, nos encontramos ante una investigación fundamentada en la construcción social del/a joven andaluz y su representación a través de aspectos relacionados con la solidaridad.

En un punto de partida inicial, la juventud se construye contradictoriamente. Se observa un abanico de opiniones que van desde el/la joven idealista y comprometido socialmente que pretende cambiar la sociedad, hasta el/la joven pasota y hedonista que no se responsabiliza de nada. Por ello analizamos en un primer momento el concepto de solidaridad de los propios jóvenes andaluces y las características que construyen socialmente a la juventud para profundizar en las prácticas solidarias que realiza la juventud andaluza junto a las motivaciones que provocan dichas prácticas.

Recalcamos la importancia de esta investigación en la utilización de la metodología cualitativa y la triangulación de los resultados con fuentes secundarias (principalmente los estudios realizados por el INJUVE y la Fundación Santa María). Debemos destacar que la mayor parte de las investigaciones dirigidas a la población juvenil española, y andaluza, se llevan a cabo mediante métodos cuantitativos. Nuestra investigación propone una recopilación de datos, un análisis y unas conclusiones, basadas en los resultados, a través de una metodología cualitativa que pone de relieve variables fundamentales, que en ciertas investigaciones sociológicas cuantitativas pasan desapercibidas, como son la construcción social de género y el estatus socioeconómico.

Por lo tanto, analizamos en esta investigación la continua construcción social de la juventud andaluza en torno a las prácticas solidarias, a partir de la dialéctica establecida entre la realidad ya construida y la realidad que estamos construyendo a través de los propios jóvenes y las personas entrevistadas que están en contacto directo con la juventud y las prácticas solidarias.

\section{Marco teórico de referencia}

En este apartado vamos a conceptualizar los términos juventud y solidaridad para poder así adentrarnos de forma unánime en la investigación.

Detengamos en primer lugar en la juventud, lo primero que debemos decir al respecto es que la juventud presenta unos límites difusos. La conceptualización de la juventud tiene un carácter ambiguo, no está claramente definida y sobretodo no están definitivamente establecidas sus demarcaciones (Wallace, y Kovatcheva, S., 1998).

"Los límites de edad no son precisos, habitualmente se considera que se inicia con la pubertad y concluye con la independencia de la tutela de la familia y la asunción de roles y status propio de la madurez, muchos de ellos relacionados con el ámbito de la producción". (Uña, O. y Hernández, A. 2004, 139).

Siguiendo la idea aportada por dichos autores, podemos decir que existe cierto acuerdo a la hora de afirmar que la juventud es una etapa de transición entre la in- 
fancia y la edad adulta1. La juventud es una "etapa de transición de la dependencia infantil a la autonomía adulta, se define por las consideraciones que la sociedad mantiene sobre ella, qué se le permite hacer, qué se le prohíbe, o a qué se le obliga". (Souto, S. 2007).

El problema, pues, surge en diferenciar y delimitar el fin de la infancia y el principio de la adultez, estas delimitaciones tan difusas son un elemento fundamental de la visión sociológica de la juventud.

Otro aspecto sobre la juventud refutado por varios autores se basa en sostener que la juventud española se ha alargado en las últimas décadas, debido a la consideración social y cultural que presenta el límite final de la juventud. Algunos autores alargan este periodo de la juventud hasta los 30 años, como opina Gómez Serrano, P.J. (1994) se deja de ser joven cuando se tiene una edad en la que se puede alcanzar la autonomía económica, se dispone de una vivienda y se puede constituir una familia. Por ello, se habla de un alargamiento de la juventud debido fundamentalmente a los/ as jóvenes que estudian y encuentran una estabilidad laboral en una edad cercana a la treintena, en el mejor de los casos.

"Los jóvenes que prolongan sus estudios se enfrentan a una posposición de su independencia económica y de su inserción social y aun así aparecen como los más privilegiados, al menos en relación al futuro". (Navarro, M. y Mateo M.J.1994:12).

Como venimos observando la juventud no se puede clasificar teniendo en cuenta únicamente aspectos cronológicos, ni biológicos, puesto que el límite entre la juventud y la adultez se basa en criterios sociales y culturales que varían considerablemente según la madurez física, social y psicológica de la persona, la educación, la incorporación al trabajo, la autonomía e independencia de cada persona que pueden desembocar en la formación de un nuevo núcleo familiar, así como la construcción de una identidad propia que es difícil de clasificar en apartados cronológicos. (Rosenmayr, L. y Allerbeck, K., 1979, 18-19).

Observemos a continuación la forma práctica con la que ciertas instituciones delimitan la juventud. Desde el ámbito institucional este tramo cronológico se determina entre los 15 y los 29 años según el Instituto de la Juventud y entre los 15 y los 24 años según los estudios de la Fundación Santa María2.

En definitiva, podemos concluir que los límites de la juventud son difusos, que dependen de factores socioculturales y que tanto en España como en los países occidentales se ha producido un alargamiento de la juventud. A pesar de ello, podemos apreciar cierto consenso que sitúa el principio de la juventud con el comienzo de la adolescencia y el final con aspectos socioculturales. De ahí que una definición ampliamente aceptada es aquella que considera jóvenes a todas las personas comprendidas entre 15 y 30 años, período en el que se desarrolla un proceso intenso

1 Aunque varios autores ponen cierta voz crítica para conseguir que la juventud no se considere como un tiempo de espera hacia el tiempo adulto. Bergua, J.A. (1999) y Montañés Serrano, M. (2000).

2 Ambas instituciones son las que más estudios han realizado sobre los jóvenes españoles. En el estudio de la fundación Santa María dirigido por González Anleo, J.M. sobre los jóvenes españoles 2010 se consideró jóvenes a las personas con edades comprendidas entre 15 y los 24 años. Mientras que en el estudio sobre jóvenes del INJUVE del año 2006 referente a juventud, solidaridad y voluntariado se consideran jóvenes a las personas con edades comprendidas entre los 15 y los 29 años. Este mismo tramo etario está considerado también por Aguinaga, J. y Comas, D. (1991) y Comas, D. et al. (2003) y Gil Calvo, E. (2004). 
de maduración física y sexual, donde se consolidan los rasgos fundamentales de la personalidad y la estructura de valores y creencias acerca del mundo.

Una vez analizada la juventud, pasamos a intentar aclarar el concepto de solidaridad. Lo primero que debemos decir respecto al término solidaridad es que es un término relativamente joven y su definición, aún hoy, resulta bastante difusa. Sin embargo dos aspectos relacionados con dicho término están bastante claros: la procedencia etimológica del latín de la palabra solidum y su origen jurídico, relacionado con la construcción. Es decir, la palabra solidaridad proviene por una parte del significado referente a lo "sólido", "compacto", "entero", "unido", "estabilidad", "firmeza"; que se vincula con la construcción y por otro lado la raíz etimológica hace referencia a la jurisprudencia. En este sentido la solidaridad se entiende como "un modo de derecho u obligación in solidum de tipo privado por lo que cada uno de los contratantes se obliga a pagar él solo la totalidad de la deuda cuando los demás miembros del pacto fallan". (Hidalgo, A., 2006, 10). Siguiendo esta vertiente Vidal, M. (1986) explica el origen etimológico de la palabra solidaridad.

"En síntesis, en la raíz etimológica de la palabra solidaridad hay dos universos significativos: el de la construcción (algo que está construido compactamente, sólidamenta) y el de la jurisprudencia (obligaciones contraídas in solidum: mancomunadamente). Del primer universo significativo quedará la lógica orgánica en el concepto de solidaridad: la unidad de un todo en el que las partes están sólidamente trabadas. Del segundo universo significativo quedará la experiencia de compartir el destino entre personas implicadas".(Vidal, M. 1996, 11-12)

En resumen, observamos con claridad la procedencia etimológica del término solidaridad, pero no se ha realizado de forma homogénea el paso del término a cuestiones sociales que son las que realmente nos interesan investigar en este trabajo. Por ello vamos a realizar una breve cronología del concepto y la utilización del término solidaridad. En cuanto a su utilización, la palabra es relativamente nueva, no aparece hasta finales del siglo XVII en francés y es a principios del siglo siguiente cuando se amplía su uso sustituyendo al término religioso caridad. A mediados del siglo XIX el término solidaridad es utilizado por el fundador de la disciplina sociológica A. Comte y se difunde al resto de idiomas europeos. Para dicho pensador la solidaridad consistía en "la unión fraternal que los individuos debían tener para salir del desorden, del caos y de la crisis social" (Comte, A., 1844). De esta forma poco a poco se deja atrás la relación de la solidaridad con la construcción y el derecho para expresar realidades sociológicas y referirse a un valor y a un principio ético.

Pero aunque Comte fue el primero en mencionar la solidaridad con un carácter eminentemente social, en sociología se entiende por solidaridad el concepto elaborado por E. Durkheim (1893) para designar al "conjunto de actitudes y comportamientos que aseguran la cohesión y la continuidad de la acción colectiva de una sociedad" (Duekheim, E., 1893).

La solidaridad, como ya hemos comentado, se ha definido de diversas maneras, veamos algunas de ellas según autores relevantes en la materia a partir de los años 90 .

"El reconocimiento práctico de la obligación natural que tienen los individuos y los grupos humanos de contribuir al bienestar de los que tienen que ver con ellos, especialmente los que tienen mayor necesidad". (De Sebastián 1996, 16)

"La solidaridad es una construcción moral edificada sobre tres dinamismos: el sentimiento compasivo, que nos lleva a ser unos para los otros; la actitud de reconocimiento, que nos convoca a vivir unos con otros, dando y recibiendo unos de otros; y 
el valor de la universalización, que nos impele a hacer unos por otros". (García Roca, J. 1998, 27).

"Por solidaridad entendemos un modo de ser y de comprendernos como seres humanos, consistente en ser los unos para los otros para llegar a estar los unos con los otros, abiertos a dar y recibir unos a otros y unos de otros". (Sobrino, J. 2002, 355).

"La identificación personal (adhesión ilimitada y total) a una causa, situación o circunstancia, persona o grupo; que implica asumir para ella beneficios y riesgos a través de la ejecución de acciones de ayuda, cooperación, colaboración individual y/o colectiva, de carácter material y/o moral, realizadas independientemente de la obligación externa, y que en su condición de necesidad interna se constituye en fuente de vivencias positivas". (CEPES, 2004, 30).

"La solidaridad, entendiéndola como un vínculo que une la sociedad y que es parte de la identidad cultural colectiva que permite establecerla como valores sociales que defienden el reconocimiento del otro con su propia identidad e individualidad generando una percepción colectiva de nosotros, implicando que cada miembro puede beneficiarse de la fuerza de los otros invirtiendo tiempo y energía en la búsqueda de un bien colectivo" (Ostau de la font, F.R. 2009, 32).

Tras el análisis de estas definiciones, observamos una concepción del término solidaridad basado fundamentalmente en una actitud moral, en un valor social. Se hace mucho hincapié en la idea de comunidad, de colectividad, en la cual cada miembro debe hacerse responsable del otro asegurando la igualdad. Los diversos autores enfocan el término solidaridad en una doble vertiente. Por un lado la solidaridad se tiene en cuenta como algo individual, moral, un valor que la persona debe tener. $Y$ por otro lado se fundamenta la solidaridad en el grupo, la comunidad, el Estado, el cual debe garantizar las relaciones recíprocas de sus miembros para que exista igualdad entre ellos.

Por lo tanto, según las definiciones expuestas, podemos sugerir que la solidaridad está ligada al mundo afectivo, moral y ético; convirtiéndose en un valor, una actitud, un modo de ser y de comprender la vida que vincula a la sociedad y la identifica con una causa: la unión de las personas para conseguir el bien colectivo.

\section{Objetivos}

\subsection{General}

- Describir la actitud y las prácticas solidarias de la población juvenil desde una perspectiva que considere a la juventud como construcción social, esto es, construida como categoría sociológica en el mismo proceso de conformación de dichas prácticas y actitudes solidarias.

\subsection{Específicos}

- Describir qué entienden por solidaridad y prácticas solidarias los/as jóvenes andaluces.

- Exponer cuáles creen que son las características que construyen socialmente a la juventud.

- Conocer si el/a joven andaluz se considera solidario.

- Analizar las prácticas solidarias de la juventud andaluza. 
- Indagar las motivaciones y las razones por las cuales los/as jóvenes andaluces realizan prácticas solidarias y la manera en que éstas dependen tanto de una determinada concepción de la solidaridad como de una autopercepción de su condición de "jóvenes".

\section{Metodología}

Para acercarnos a nuestro objeto de estudio y poder lidiar con los objetivos planteados, vamos a utilizar fundamentalmente la metodología cualitativa. En este sentido nuestro objeto de estudio se sustenta en un valor: la solidaridad. Pensamos que las características de la metodología cualitativa se ajustan mejor que las cuantitativas a la hora de investigar un concepto como el de solidaridad, debido fundamentalmente a la dificultad existente a la hora de profundizar en el estudio de un sentimiento, un valor o una actitud si no es a partir de las declaraciones o verbalizaciones subjetivas de los sujetos. Como estamos interesados, por otro lado, en el proceso de circulación social y construcción de los discursos que incluyen categorías como "joven", "juventud" o "solidaridad", hemos optado por este conjunto de técnicas cualitativas de investigación que, no obstante, se complementarán con un uso extensivo de las distintas fuentes secundarias de datos estadísticos sobre la población joven en España.

Las técnicas de investigación cualitativa que hemos utilizado a lo largo de esta investigación son la entrevista en profundidad y el grupo de discusión. Ambas técnicas se complementan muy bien entre sí, nos aportan información dotándonos de un marco de análisis discursivo propio para conseguir los objetivos planteados en este trabajo.

A la hora de seleccionar los/as informantes para las entrevistas personales hemos decidido entrevistar tanto a jóvenes como a personas adultas que se encuentran en contacto directo con la juventud y se hallan implicados en el ámbito solidario juvenil institucional, al participar en entidades de gestión de la práctica solidaria. La forma de seleccionar a los entrevistados/as viene argumentada por la selección previa de ciertos informantes y la consecuencia del llamado efecto bola de nieve. La muestra final de las personas entrevistadas ha sido de 16 personas, ocho hombres y ocho mujeres, con una edad comprendida entre los 25 y los 60 años.

Hemos elegido la cantidad ecuánime de informantes respecto a hombres y mujeres para añadir a nuestra investigación un enfoque y diseño sensible a la cuestión de género. Respecto a cuantas entrevistas debemos realizar nos basamos en el muestreo por saturación teórica. En cuanto a la representatividad hemos decidido elaborar un eje de coordenadas teniendo en cuenta dos variables fundamentales para la obtención de información en referencia al objeto de estudio de la investigación: la naturaleza de la práctica solidaria (pública o privada) y el estatus profesional del entrevistado (alto o bajo).

Las variables se fundamentan en los siguientes criterios: personas vinculadas a la práctica solidaria institucional en los jóvenes dependiendo si la entidad a la que pertenecen es pública o privada y la naturaleza de la implicación de los/as informantes en las propias entidades atendiendo a su posición dentro de la misma o a su nivel jerárquico (estatus profesional).

Además dentro de estas variables hemos mantenido una muestra equitativa respecto al género y la geografía andaluza (suponemos dos submuestras, Andalucía oriental y occidental). El siguiente gráfico y el siguiente cuadro muestran la selección y la representatividad de los informantes elegidos/as para realizar las entrevistas. 
Figura 1: muestra informantes entrevistados.

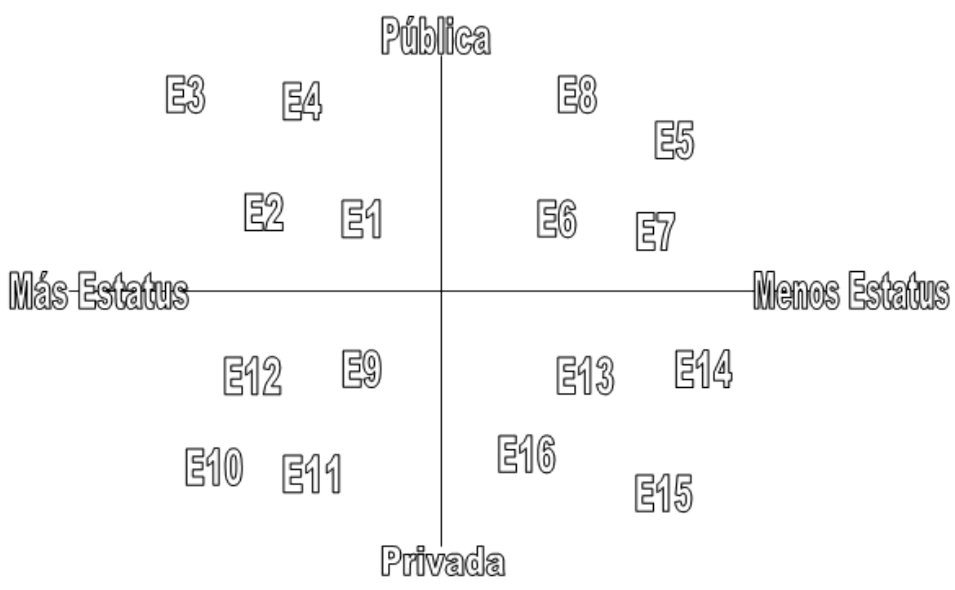

Cuadro 1: Perfil de los informantes entrevistados.

\begin{tabular}{|c|c|c|c|c|}
\hline & Relación práctica solidaria y juventud. & Status social & Sexo & Edad. \\
\hline E.1. & Observatorio voluntariado. & Alto & Mujer & 35 \\
\hline E.2. & Voluntariado Universidad. & Medio & Mujer & 30 \\
\hline E.3. & Asociación juvenil. & Medio & Mujer & 28 \\
\hline E.4. & Asociación solidaria. & Alto & Hombre & 40 \\
\hline E.5. & Asociación juvenil. & Medio bajo & Mujer & 25 \\
\hline E.6. & Voluntario juvenil. & Medio & Hombre & 43 \\
\hline E.7. & Técnica de juventud pueblo. & Medio bajo & Mujer & 34 \\
\hline E.8. & Técnica Juventud ciudad. & Medio & Mujer & 39 \\
\hline E.9. & ONG Juvenil. & Medio & Hombre & 23 \\
\hline E.10. & Voluntariado. & Alto & Hombre & 37 \\
\hline E.11. & Plataforma voluntariado. & Alto & Hombre & 33 \\
\hline E.12. & ONG juvenil. & Alto & Mujer & 34 \\
\hline E.13. & Voluntaria de una ONG. & Bajo & Mujer & 21 \\
\hline E.14. & Movimientos sociales. & Bajo & Hombre & 38 \\
\hline E.15. & Profesor Educación Secundaria. & Medio & Hombre & 50 \\
\hline E.16. & Voluntaria asociación. & Mujer & 25 \\
\hline
\end{tabular}

Fuente: elaboración propia.

Por su parte, los grupos de discusión se formaron en torno a dos variables fundamentales para la investigación: el estatus socioeconómico y el hábitat, divididas ambas por los siguientes grupos de edad, desde los 15 años hasta los 22 y desde los 23 hasta los 29. Dichas variables son tomadas como ejes fundamentales teniendo en cuenta además en todos los grupos la equidad respecto al género y la representatividad de la geografía andaluza. En total se realizarán 8 grupos de discusión, cuatro de ellos realizados en Andalucía occidental y los otros cuatro en Andalucía oriental. La siguiente figura muestra dicha distribución. 
Figura 2: muestra grupos de discusión.

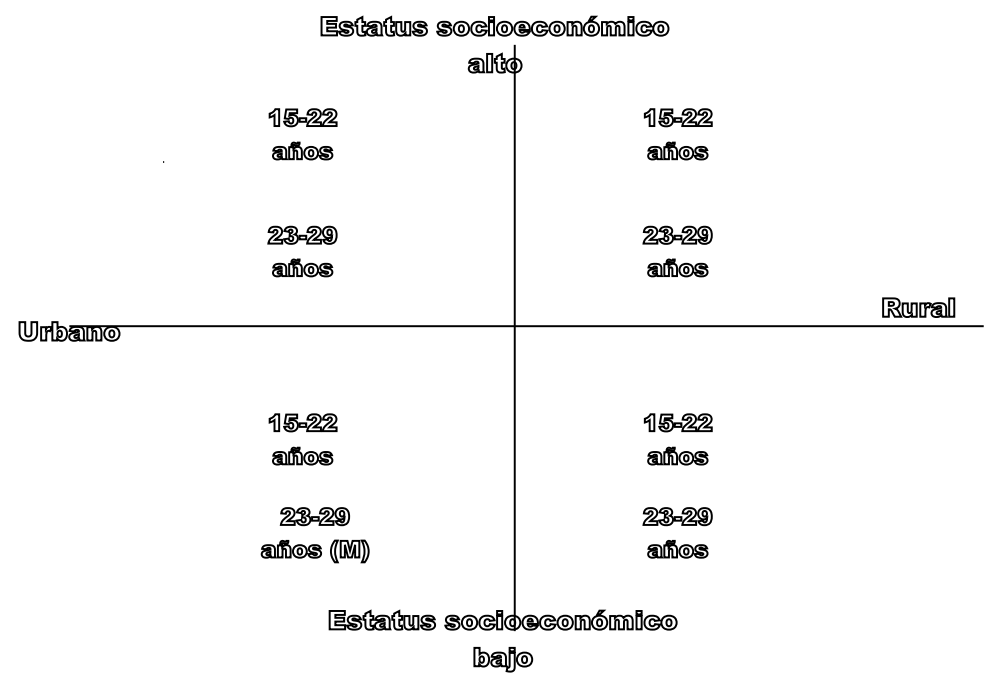

Al igual que en las entrevistas, los/as informantes fueron elegidos siguiendo la bola de nieve que nos proporcionaban las entrevistas y la posibilidad de reunir a distintos jóvenes con los perfiles seleccionados, teniendo en cuenta la perspectiva de género y terminando dicha selección mediante el proceso de saturación teórica.

Respecto a los temas tratados tanto en las entrevistas como en los grupos de discusión presentamos el siguiente cuadro donde aparece el guion orientativo para llevar a cabo ambas técnicas de investigación cualitativa en referencia a los objetivos planteados.

Cuadro II: guion general utilizado en las entrevistas y los grupos de discusión.

\begin{tabular}{|c|}
\hline GUION ORIENTATIVO. \\
\hline Breve presentación del objetivo de la entrevista/grupo de discusión. \\
\hline Características sociodemográficas o de encuadre biográfico. \\
\hline ¿Qué entiende por solidaridad y prácticas solidarias? \\
\hline ¿Son solidarios los jóvenes españoles? ¿Cuáles cree que son las características fundamentales de la \\
juventud?
\end{tabular}

Fuente: elaboración propia

Como hemos mencionado con anterioridad la metodología utilizada en esta investigación consiste en una triangulación metodológica donde combinamos recopilaciones de datos primarios construidos a partir de técnicas cualitativas con datos cuantitati- 
vos de carácter secundario, provocando una interacción entre ambas fuentes y entre ellas con el marco teórico. Para este cometido, principalmente, nos apoyaremos en los resultados obtenidos en los estudios del INJUVE y la Fundación Santa María.

Hemos tenido en cuenta las variables utilizadas por estas investigaciones y cruzado los datos analizando el discurso del análisis cualitativo con los resultados cuantitativos ya obtenidos, teniendo en cuenta la perspectiva temporal.

Por último mencionamos que para el análisis de los datos obtenidos en las entrevistas y los grupos de discusión nos hemos ayudado de la herramienta informática Atlas.ti. Dicho programa informático nos facilita un análisis de los datos sustentado en la Teoría fundamentada, por ello hemos considerado que la utilización de Atlas.ti para nuestra investigación nos puede ayudar a la hora de otorgarle a nuestros resultados mayor rigor y objetividad.

Siguiendo pues las aportaciones de la teoría fundamentada al análisis cualitativo surge el programa informático Atlas.ti. Mediante dicho programa hemos creado una unidad hermenéutica denominada "Solidaridad y juventud", dentro de ella hemos insertado los documentos, concretamente las transcripciones realizadas de las dieciséis entrevistas y los ocho grupos de discusión. Una vez insertados los documentos primarios los hemos codificado a través de de citas-códigos realizando así una primera criba, obteniendo una reducción de los datos brutos y progresando en distintos niveles de abstracción analítica a partir del discurso como dato textual.

Siguiendo los principios de la teoría fundamentada, hemos creado códigos con un nivel mayor de abstracción que coinciden con nuestros objetivos. Presentamos a continuación la agrupación de los supercódigos: concepto de solidaridad, concepto y características de la juventud, ¿son solidarios los jóvenes andaluces?, estatus socioeconómico, hábitat, motivaciones y prácticas solidarias.

\section{Resultados}

\subsection{El concepto de solidaridad de los jóvenes andaluces}

En primer lugar vamos a mostrar el concepto de solidaridad, según las ideas aportadas en el marco teórico, para después comprobar su relación con el concepto de juventud que tienen los jóvenes andaluces.

En el marco teórico hacemos referencia al concepto de solidaridad desde autores contemporáneos hacia diferentes conceptos elaborados por los autores clásicos de la sociología. Como hemos señalado, los autores contemporáneos marcan la solidaridad en los siguientes términos: ayuda recíproca, construcción moral dirigida hacia los que tienen mayor necesidad, el objetivo es el bienestar de la comunidad, dar y recibir los unos con los otros, colectividad, acción conjunta, la identificación personal a una causa, situación o circunstancia, persona o grupo; la unión de personas para conseguir un fin, el vínculo que une a la sociedad y pertenecer al mundo afectivo, moral y ético.

Por parte de la sociología el término solidaridad se vincula fundamentalmente a Durkheim que matiza este término como "el conjunto de actitudes y comportamientos que aseguran la cohesión y la continuidad de la acción colectiva de una sociedad" (Durkheim, 1893). Según dicha definición, la solidaridad tiene un fin en sí misma que es mantener la unión del grupo y se realiza dentro de una sociedad concreta hacia el engrupo, no hacia el exogrupo. 
En el marco teórico diferenciamos los aspectos en los que los autores se centran para abordar el concepto de solidaridad. Estos aspectos hacen referencia al agente objeto de la solidaridad dentro del endogrupo ya sea la familia, el grupo de iguales, la comunidad, personas de la misma clases social, etc. Aunque también siguen la estela de Durkheim marcando la solidaridad como un hecho de cohesión social.

A continuación vamos a mostrar lo que los jóvenes españoles entienden por solidaridad tal y como ha quedado representado este concepto en su discurso. Ya conocemos, en parte, lo que los/as jóvenes opinan al respecto cuando han sido consultados mediante otro tipo de instrumentos de observación. Por ejemplo, según el sondeo realizado en 2006, a nivel nacional, por el INJUVE sobre juventud, solidaridad y voluntariado "la mayor parte de los jóvenes (52\%) se muestran de acuerdo con la afirmación: solidaridad es tener en cuenta a los demás" (INJUVE, 2006). Con ello podemos apreciar que la juventud se fundamenta en una idea basada en el otro; en primer lugar se determina que la solidaridad se dirige hacia personas y que para ejercer dichas prácticas basta con tener en cuenta a las personas hacia las que se dirige dicha solidaridad.

Los datos obtenidos en nuestra investigación señalan que los/as jóvenes andaluces muestran una visión de la solidaridad relacionada más con términos contemporáneos como ayudarse los unos a los otros, compartir o tener empatía. Sin embargo no mantienen la visión de Durkheim de establecer la solidaridad como la unión de un grupo sino más bien como la ayuda desinteresada a todas las personas. Esta afirmación es bastante lógica puesto que el concepto de Durkheim no es general, sino que aspira a convertirse en categoría sociológica para explicar ciertos fenómenos sociológicos. Durkheim no tiene interés en describir ningún movimiento solidario, más bien pretende retratar sociológicamente las ligas existentes entre individuos en un contexto social.

Por otra parte sí podemos reafirmar la vinculación de los datos obtenidos con los jóvenes españoles encuestados en 2006 puesto que la solidaridad se basa en una relación de ayuda recíproca hacia los demás de forma desinteresada, cómo analizamos a continuación.

En nuestra investigación, el concepto de solidaridad que se repite de forma abrumadora es aquel que plantea que la solidaridad es ayudar sin recibir nada a cambio. En torno a esta idea se barajan otras posibilidades dentro del discurso del joven andaluz como que la solidaridad es ayudar, ponerse en el lugar del otro, compartir o hacer algo por los demás. Por lo tanto podemos decir que para el/a joven andaluz el concepto de solidaridad se enfatiza en la ayuda hacia otras personas, compartiendo, siendo empáticos y sin recibir por ello nada a cambio. Debemos mencionar también que en este último aspecto se crea un discurso en el que se reflejaba la posibilidad de recibir algo a cambio pero que no fuese material, como por ejemplo la satisfacción personal de ayudar a otra persona.

De forma más minoritaria se han planteado también otros aspectos como que la solidaridad es cambiar la sociedad, ser altruista, tener cohesión social, dar tu tiempo, buscar la igualdad, obtener justicia o hacer feliz al otro. Además del discurso plasmado por los jóvenes andaluces debemos tener en cuenta la ausencia del discurso, aquello que no se dice, en este caso es oportuno anotar como el concepto de solidaridad de los/as jóvenes se refiere en la mayor parte de los casos a una solidaridad puntual que no compromete al joven y que subsiste de forma caritativa en lugar de una solidaridad transformadora. 
Para corroborar dicha información presentamos los siguientes fragmentos de las transcripciones de los entrevistados y entrevistadas donde se refleja la visión general de la solidaridad entendida como "ayudar sin recibir nada a cambio":

"Las prácticas solidarias son aquellas que tienen una finalidad desinteresada, sin esperar una remuneración, una recompensa a posteriori. Sí es cierto que hay una recompensa, pero es una recompensa de haber hecho lo que tú realmente quieres hacer con ese servicio". (Hombre, 30 , nivel socioeconómico medio bajo: movimientos sociales).

"Lo que yo entiendo por solidaridad es ofrecer tus servicios sin esperar nada a cambio". (Mujer, 21, nivel socioeconómico medio bajo, voluntaria).

"Yo entiendo la solidaridad como hacer algo sin recibir nada a cambio, algo que tú haces porque quieres ayudar a las personas, que lo ves gratificante y te sientes bien con eso". (Mujer, 25, nivel socioeconómico alto, hábitat rural).

Siguiendo esta dinámica la solidaridad se vincula con la idea de ayudarse los unos a los otros, con compartir y con tener empatía, como podemos apreciar sucesivamente en los siguientes fragmentos:

"Yo creo que solidaridad es la ayuda que prestamos a otras personas $u$ otras personas nos prestan a nosotros, el ayudarse mutuamente". (Hombre, 28, nivel bajo, Hábitat rural).

De nuevo se vincula la solidaridad con el hecho de ayudar a personas pero no con el fin de mantener una cohesión social.

"Solidaridad es que todo lo que tengas tú lo compartas con los demás, si tienes algo compartirlo con amigos o personas que lo necesiten". (Mujer, 27, nivel alto, Hábitat urbano).

Como se muestra anteriormente los/as jóvenes entienden la solidaridad como un aspecto moral que se dirige personalmente hacia personas concretas.

"El ser empático, el ser capaz de ponerte en la piel del otro, saber responder siempre de una manera asertiva, y el saber y el querer poder ayudar en cualquiera de tus posibilidades". (Mujer, 25, nivel socioeconómico medio bajo: asociación juvenil).

Siguiendo la idea personal de ayudar al otro, se muestra una idea, moral, ética e individual que genera la comprensión de la situación de otras personas.

En definitiva tenemos una definición clara del/a joven andaluz que habla de ayudar a los demás sin recibir nada a cambio entendida desde una determinación personal dirigida a personas concretas, basada en aspectos morales como el compartir, la empatía, el ayudar, etc. Esta definición coincide con la aportada por los diferentes estudios 3 que definen la solidaridad como tener en cuenta a los demás, coincide en parte con lo aportado por los autores contemporáneos que mezclan la vinculación ética y moral con la cohesión social.

Respecto a las influencias de las variables dependientes cabe destacar la tendencia de vincular la solidaridad con la caridad por parte de los jóvenes de estatus socioeconómico alto frente a los jóvenes con un nivel socioeconómico bajo que relacionan el término solidaridad con el término justicia.

3 Entre ellos INJUVE (2006) y Fundación Santa María (2010). 
"La justicia es la forma de entender la solidaridad, desde mi punto de vista si no hay justicia no hay solidaridad". (Hombre, 28, nivel socioeconómico bajo: movimientos sociales).

"Entiendo que ayudar a los demás es darles a aquellas personas que no tienen nada para poder ayudarles". (Mujer, 25, nivel socioeconómico alto, urbano).

Por lo tanto nos encontramos con una definición de solidaridad que remarca los siguientes aspectos de la solidaridad: empatía, hacer por los demás.ayudarse los unos a los otros, compartir y ayudar sin recibir nada a cambio.

\subsection{Características de la juventud}

La visión de los jóvenes andaluces y de los expertos entrevistados muestra una apreciación negativa de la juventud, sobre todo por la propia juventud y por los jóvenes de mayor edad. Minoritariamente se piensa que la juventud tiene aspectos positivos como que es reivindicativa, activa y posee un espíritu crítico, pero mayoritariamente el/a joven se clasifica fundamentalmente como hedonista, egoísta y pasota. Las evidencias que mostramos a continuación resumen la apreciación que los jóvenes tienen sobre ellos mismos:

"Yo creo que la juventud es más pasota, es una época un poco de pasar, hay gente que se preocupa pero a la mayoría le da igual lo que le pase al mundo". (Hombre, 27, nivel socioeconómico bajo, rural).

"Somos egoístas, primero piensas por ti mismo y luego por los demás". (Mujer, 17, nivel socioeconómico bajo, rural).

"Ahora en general creo que la juventud es más pasota, creo que ahora mismo los jóvenes tienen otros objetivos, aunque también depende del ámbito en el que te muevas, si lo enfocas a lo social sí, porque es básico ser solidario, si no cada uno va a su bola". (Mujer, 25, nivel socioeconómico bajo, voluntariado).

"Yo creo que la juventud es pasota, les da igual todo, aunque ese pasotismo puede ser por desconocimiento de las cosas. Lo que yo pienso también es que te dejas llevar por otras personas. No todos los jóvenes son pasotas pero la mayoría sí". (Hombre, 28, nivel socioeconómico alto, rural).

Como podemos observar la percepción de la juventud andaluza sobre sí misma se valora en relación a factores negativos coincidiendo con las ideas que visualizan al joven como hedonista y narcisista (Revilla, 2001:107). En el discurso se aprecia por tanto una construcción social negativa del/a joven andaluz, son muy pocos los que consideran que la juventud es un agente de cambio social, que los jóvenes son inconformista y reivindicativos, frente a un discurso que los considera egoístas, pasotas, despreocupados, etc.

Las variables en este aspecto no son significativas, el joven es construido con valores negativos referentes al hedonismo y al pasotismo independientemente de la edad, el estatus socioeconómico y el género.

Como podemos, la construcción social de la juventud por parte de los jóvenes andaluces se enfoca desde un punto de vista negativo. Debido a que los aspectos señalizados como característicos de los jóvenes son los siguientes: despreocupados, pasotas, egoístas, impulsivos, dormidos.Sin embargo, en el discurso aparece además la idea previa de que el/a joven es el motor del cambio y por lo tanto debe ser impulsivo, inconformista, revolucionario. Este planteamiento se genera fundamental- 
mente por las personas entrevistadas que trabajan en contacto con la juventud en prácticas solidarias.

Por lo tanto, dialogando con los datos, podemos atisbar la construcción de la juventud según planteamientos diversos que se crean en función de la conveniencia de quien los genera. Algunas veces se construye la juventud de forma positiva y otras veces mediante aspectos negativos, todo ello segregando un conjunto de población al que se dota de vida propia al margen del resto de la sociedad. A pesar de ello es abrumadora la percepción negativa que tiene la propia juventud andaluza sobre sí misma, seguramente en función del reflejo de la opinión de los adultos que por una parte idealizan la juventud otorgándole a los/as jóvenes el papel de impulsores inconformistas propulsores del cambio de una sociedad, mientras que por otra parte los visualizan desde una construcción negativa tachándolos de irresponsables, pasotas, inexpertos, egoístas, etc.

Para finalizar este apartado nos fijamos en el eje discursivo de nuestra investigación en referencia a la construcción positiva o negativa de la juventud andaluza. Se aprecia en el discurso cierta diferenciación entre las opiniones de los/as profesionales que trabajan en contacto directo con la juventud y los/as propios jóvenes, dejando una apreciable segmentación a la hora de construir la juventud desde aspectos positivos o negativos. En referencia a esta apreciación tomamos como dato de especial relevancia la tendencia de los/as profesionales a mantener una construcción de la juventud basada en aspectos positivos, siendo la percepción de los/as jóvenes una construcción más negativa de su propio entorno juvenil.

\section{3. ¿Son solidarios los jóvenes andaluces?}

Para responder a esta pregunta analizamos el discurso tanto de los jóvenes como de los expertos teniendo en cuenta las diferentes variables y la respuesta es bastante clara: la juventud no es considerada como solidaria. Si nos fijamos que en el apartado anterior la visión del joven se construye alrededor de aspectos negativos era de esperar que el/a joven se construya de forma coherente como no solidario. La mayor parte de los/as jóvenes cree que la juventud no es solidaria y aumenta dicha consideración conforme avanza la edad.

Podemos observar que los jóvenes ven en la solidaridad un hecho excepcional, minoritario y que no alcanza al conjunto del colectivo, tal y como muestran algunas de sus intervenciones durante nuestras entrevistas y grupos de discusión.

"Son muy pocos los que ayudan, si tuviera que decir un porcentaje diría que un $30 \%$ o menos". (Mujer, 23, nivel socioeconómico alto, rural).

"Creo que los jóvenes no son solidarios, porque ni los medios de comunicación, ni los juegos son solidarios. El proceso que tienen ahora los jóvenes para estar con otros jóvenes no les implican tener valores solidarios, no lo tienen asimilado". (Mujer, 29, nivel socioeconómico bajo, urbano).

"Yo diría que menos, no llegará ni a un 10\% los jóvenes solidarios, realmente no ayuda casi nadie, nos movemos siempre por algo". (Hombre, 26, nivel socioeconómico alto, rural).

"Visto desde fuera diría que los jóvenes no son solidarios, actualmente hay muy poco porcentaje de jóvenes que realicen prácticas solidarias". (Hombre, 37, nivel socioeconómico alto, voluntariado). 
Por otra parte debemos decir que los informantes relacionados con asociaciones solidarias valoran positivamente la participación de la juventud en actividades solidarias aunque piensen que esa juventud participativa es minoritaria.

"La gente que viene aquí suele ser gente con muchas ganas de moverse, mucho entusiasmo, gente súper activa, que no quiere parar, gente muy emprendedora, muy enriquecedora que va de una parte a otra del mundo, que lo recorre todo, quieren aprender y ayudar". (Mujer, 25, nivel socioeconómico medio, asociación juvenil).

"Sí, mis amigos si son solidarios porque gran parte de nuestro grupo de amigos somos de aquí de la asociación, entonces, pero los que no son de aquí no mucho". (Hombre, 16, nivel socioeconómico alto, urbano).

También se remarca el discurso que habla de una juventud solitaria minoritaria pero de jóvenes que realizan muchas actividades a la vez, es decir, se muestra una juventud insolidaria pero destacan ciertos jóvenes que son solidarios, sacan buenas notas, tienen una ideología clara, participan en todo tipo de actividades, etc.

"El problema suele ser que la gente que tiene mayor compromiso político solidario es la gente que al final termina estando en más cosas; con lo cual es mentira esa idea de que los jóvenes no hacemos prácticas solidarias porque no tenemos tiempo, no, perdona, la gente que quiere hacer hace y hace cosas y se saca su carrera y hace no sé cuánto, es decir suele ser al contrario, quien sólo estudia es porque no tiene mayor interés que hacer eso". (Hombre, 38, nivel socioeconómico alto, asociación solidaria).

Los datos obtenidos coinciden con los datos cuantitativos del estudio del INJUVE (2006), en dicha investigación también se muestra un joven poco solidario, aunque no hay datos concretos sobre prácticas solidarias de forma general, sí muestra el desinterés de los jóvenes a la hora de realizar prácticas voluntarias. Según este sondeo "aparece una mayoría de jóvenes (72\%) que declara no haber colaborado nunca con organizaciones de voluntariado". (INJUVE, 2006).

A pesar de lo comentado sí hay jóvenes que realizan prácticas solidarias por ello vamos a exponer a continuación los contextos donde se llevan a cabo dichas prácticas.

\subsection{Las prácticas solidarias de la juventud andaluza}

En este apartado analizamos las prácticas solidarias de los jóvenes andaluces, además, manteniendo la línea de nuestros objetivos, observamos la coherencia discurso-práctica de nuestros informadores, en referencia a la solidaridad.

En primer lugar nos planteamos si las prácticas solidarias que realizan los jóvenes andaluces se realizan de forma puntual y caritativa o sin embargo son prácticas transformadoras que suponen un compromiso a largo plazo. Pues en consonancia con los resultados anteriores la construcción del/a joven solidario se inserta dentro de una juventud que realiza pocas prácticas solidarias y las que realizan son fundamentalmente caritativas y puntuales.

"Yo creo que la mayoría de las prácticas que realizan los jóvenes son asistenciales porque pienso que no se tiene el concepto de solidaridad como una herramienta para la transformación social sino como hacer una acción que es buena pero que no llega al fondo del problema”. (Mujer, 18, nivel socioeconómico medio alto, rural).

"De largo la solidaridad caritativa está mejor vista y se pone más en práctica con cuestiones de la campaña del kilo en Navidad, con cuestiones de una catástrofe natural en cualquier sitio, ahí están. Denunciar y ponerse manos a la obra bajo situa- 
ciones de justicia cercanas que nos permita mirarnos a nosotros mismos y decir que tendría que cambiar yo de lo que yo hago, de lo que hacen mis vecinos para que la injusticia que nos rodea no se produzca o intentar evitarla está mucho más limitado". (Hombre, 35, nivel socioeconómico medio bajo, movimientos sociales).

En segundo lugar exponemos los principales sectores donde la juventud andaluza realiza prácticas solidarias. En la mayoría de los discursos destacan dos colectivos fundamentales hacia donde se dirige la solidaridad del joven andaluz: la participación en actividades solidarias con menores y con personas mayores.

"Normalmente suelen participar con personas mayores, con niños pequeños, o en eventos puntuales de todo tipo. Últimamente se mueven también por temas de alimentación, muchos jóvenes que están trabajando en comedores sociales, con los bancos de alimentos, etc. Y también me consta que en las capitales como Málaga y Granada son muchos los jóvenes que colaboran en atender a personas que se encuentran sin hogar, sobretodo en invierno". (Hombre, 50, nivel socioeconómico medio, profesor).

Junto a estos colectivos destacan prácticas solidarias puntuales como donar ropa o participar con un banco de alimentos. Además se menciona de forma alternativa la participación de los jóvenes en actividades relacionadas con la cooperación internacional, el medio ambiente, personas sin hogar y personas inmigrantes.

"Normalmente pues dar ropa, comida a gente que no tiene o que le hace mucha falta y se le ayuda". (Mujer, 28, nivel socioeconómico bajo, rural).

"Las prácticas solidarias de los jóvenes, según mi experiencia, se dirigen hacia colectivos desfavorecidos, fundamentalmente hacia personas sin hogar, estar en comedores repartiendo comida, incluso en la calle. Les gusta el contacto directo, el trabajo de campo". (Mujer, 25, nivel socioeconómico bajo, voluntaria asociación solidaria).

"Creo que los jóvenes son bastante solidarios en actividades de voluntariado sobre todo con personas mayores, también en el ámbito del medio ambiente o personas que estén en riesgo de exclusión social como los inmigrantes". (Hombre, 15, nivel socioeconómico medio alto, rural).

Los datos obtenidos en el discurso mantienen cierta discrepancia con los datos cuantitativos ofrecidos por el estudio del INJUVE (2006) que muestra como principal práctica solidaria "tener conversaciones con sus amigos o compañeros, con mayor o menor frecuencia a lo largo del año, sobre países o personas en situación de necesidad".

Además de esta controversia, planteamos la duda sobre si conversar con amigos es una práctica solidaria. Desde la conceptualización del término solidaridad analizado en el marco teórico la conversación sobre personas en situación de necesidad no plantea una ayuda en sentido práctico, es decir no sería una práctica solidaria, aunque puede entrar dentro de la sensibilización y del sentimiento de empatía que se remarca por los jóvenes andaluces a la hora de definir la solidaridad.

Dicho estudio apunta que las principales actividades solidarias llevadas a cabo por los jóvenes son aquellas que se realizan de forma puntual, manteniéndose así una coherencia con el discurso obtenido en esta investigación que identifica la solidaridad con una práctica esporádica. Concretamente describe la donación de dinero para países o personas que lo necesitan (48\% de las los jóvenes encuestados realizan actividades de este tipo) y la colaboración más o menos puntual en acciones solidarias (ayuda a los vecinos, protección de la naturaleza, etc.) un 39\%. 
Pero si entendemos la práctica solidaria como voluntariado la coherencia con los datos aportados por el estudio del INJUVE (2006) es muy significativa, según el sondeo "las actividades de voluntariado más interesantes para los jóvenes son aquellas relativas a la infancia y la juventud (14\% de los jóvenes encuestados realizan prácticas con este colectivo), la ayuda al Tercer Mundo y países en conflicto (11\%), el trabajo con discapacitados (11\%), la ecología y el medioambiente $(10 \%)$ y las relativas a la salud y la sanidad (9\%). Les siguen las actividades de voluntariado con ancianos (8\%), con drogodependientes y alcohólicos (7\%), de ayuda a la pobreza (7\%) y el trabajo con inmigrantes (6\%)".

Por lo tanto podemos relacionar el discurso creado mediante nuestros informantes con los datos aportados por los estudios del INJUVE. Si realizamos una lista entre ambos las prácticas solidarias que realizan los jóvenes serían las siguientes: menores, infancia, personas mayores, donar ropa, donar comida a un banco de alimentos, cooperación internacional, ayuda al tercer mundo, medio ambiente, ecología, personas sin hogar, personas inmigrantes, ayudar a los vecinos, personas con discapacidad, sanidad y drogodependencia.

Además este estudio coincide en hacer significativa la elección de prácticas solidarias por parte de los jóvenes en función del género, destacando que las mujeres jóvenes realizan actividades relacionadas con el cuidado de personas y los hombres fundamentalmente actividades medioambientales.

El estudio del INJUVE es claro, en este aspecto concluye que "los chicos y las chicas jóvenes muestran diferencias significativas en cuanto a su interés por las actividades de voluntariado. Las preferencias de los hombres jóvenes se concentran en actividades de ecología y medioambiente; mientras que las mujeres en mayor medida que los hombres se decantan por el apoyo a la juventud, la salud y la ayuda a los ancianos". (INJUVE, 2006).

En nuestra investigación el discurso fluye de manera similar, para corroborar esta idea plasmamos la evidencia de un profesional que trabaja en contacto directo con la juventud.

"Principalmente realizan actividades con personas mayores, o con niños y juventud con problemáticas, aunque sobre todo las mujeres, bueno los hombres también pero en otro tipo de actividades más de fuerza o también medioambientales, sí muchos jóvenes trabajan en cuestiones medioambientales y ellas más en el cuidado de las personas". (Mujer, 34, nivel socioeconómico medio, técnica de juventud).

Por lo tanto ambas investigaciones coinciden en señalar las siguientes prácticas como las principales prácticas a las que se dedica la juventud: menores, personas mayores, ayuda al tercer mundo, medio ambiente y en menor medida ayudar a colectivo de inmigrantes.

\subsection{Motivaciones de los jóvenes andaluces para realizar prácticas solidarias}

Por último exponemos los resultados en torno a las motivaciones de los/as jóvenes andaluces para realizar prácticas solidarias. Dentro de las motivaciones declaradas para realizar prácticas solidarias nos encontramos principalmente con aspectos que no se relacionan directamente con acciones altruistas. Las principales razones por las que los jóvenes andaluces realizan acciones solidarias (también entendidas dentro del voluntariado), a tenor de cómo estas han sido verbalizadas en el espacio de nuestro dispositivo de investigación por parte de los y las entrevistados son:

- Poder insertarse de cierta forma en el mercado laboral. 
- Porque un amigo le ha incitado a realizar ciertas prácticas solidarias y de esta forma aumentan sus relaciones sociales.

- Ayudar a gente porque ellos, o alguna persona cercana, han pasado por una situación similar a la que sufre la persona objeto de la solidaridad.

En el discurso se pueden apreciar motivaciones tan distantes como encontrar un trabajo o cambiar el mundo. Al analizar los datos quedan patentes dos tipos de motivaciones, aquellas que, en cierta manera, benefician al propio sujeto solidario y otras que son más altruistas. Cabe preguntarse pues si la práctica solidaria es aquella que se lleva a cabo sin recibir nada a cambio, como se plantea en la conceptualización de la solidaridad, puesto que la mayoría de las motivaciones por las cuales los jóvenes andaluces realizan prácticas solidarias incluyen un cierto interés personal. Sobre todo los jóvenes de edad más avanzada piensan que concretamente el voluntariado les puede servir para introducirse en el mundo profesional e ir probando su perfil académico en busca de un posible trabajo.

"Bueno aquí se ha hablado un poco de las motivaciones que tienen los voluntarios y últimamente, claro puesto que no encuentran trabajo, pues su motivación es esa principalmente, ir a una entidad con el fin de quedarse trabajando". (Mujer, 35, nivel socioeconómico medio alto, observatorio voluntariado).

Por otra parte, fundamentalmente los jóvenes de menor edad, creen que las acciones solidarias pueden servir para aumentar el capital social y las relaciones interpersonales de los jóvenes que las realizan. El punto de arranque para iniciar dichas actividades es tener amigos que ya realicen dichas prácticas creando así una especie de moda solidaria o de cultura de la solidaridad que mueve a ciertos sectores de la juventud.

"Yo creo también que participan porque establecen relaciones sociales, vínculos fuera de lo que es el ocio nocturno típico". (Hombre, 15, nivel socioeconómico medio alto, rural).

Este planteamiento es corroborado por los datos que ofrece el INJUVE (2006) que señala que relacionarse con otras personas es una motivación fundamental para los jóvenes a la hora de realizar prácticas solidarias.

Durante los discursos han surgido otras motivaciones, unas siguiendo la línea anterior y otras con carácter más altruista. Las principales son: cambiar las acciones injustas, sentirse útiles, satisfacción personal, empatía y vivir experiencias. Las principales razones como son sentirse útiles y relacionarse con otras personas coinciden con las motivaciones que aporta el sondeo del INJUVE (2006), sin embargo existe discrepancia con los datos de dicho sondeo que remarca como principales motivaciones de los jóvenes a la hora de realizar actividades solidarias la realización de un servicio social a la comunidad $(87 \%)$, las creencias morales $(81 \%)$ y las creencias religiosas $(60 \%)$.

Es decir, mientras nuestra investigación aporta datos que dirigen nuestras conclusiones acerca de unas motivaciones egoístas por parte de los jóvenes andaluces para realizar prácticas solidarias (en concordancia con la construcción social negativa de las características de la juventud), el estudio del INJUVE muestra una juventud solidaria por convencimiento, ya sea para mejorar la comunidad o por dictamen de creencias religiosas o ético-morales. 


\section{Análsis de los resultados}

Una cuestión clave emanada del análisis del discurso juvenil es lo relativo a la conceptualización del término solidaridad por parte de la juventud andaluza, que se fundamenta en una idea básica que pone de manifiesto el hecho de que la solidaridad es "ayudar a los demás sin recibir nada a cambio". Conceptualización distintiva que vincula el concepto con determinadas representaciones del hecho solidario tratadas en el marco teórico de este mismo documento. ¿De qué manera se evidencia el concepto en la práctica discursiva de los informantes? En este sentido cabría esperar que las motivaciones del/a joven andaluz/a versaran sobre actitudes e ideas solidarias que concuerden con la coletilla "sin recibir nada a cambio", es decir, desinteresadas. Sin embargo, las motivaciones declaradas muestran cierta contradicción puesto que, sobre todo para los informantes de más edad, la principal causa para realizar actividades solidarias es la posible inserción al mercado laboral. A decir de los más jóvenes, en cambio, la razón principal por la que realizan prácticas solidarias es referente a las redes de sociabilidad de las que disponen (y disfrutan), es decir, porque un amigo las realiza, posibilitando el aumento de las relaciones sociales. Dichas motivaciones, no obstante, concuerdan con el contexto social actual donde se sitúa la juventud (pero también el mundo adulto). Es decir, aunque entienden que la solidaridad es otra cosa, el/la joven andaluz, particularmente los de una edad superior, muestra una inquietud preexistente a la verbalización sobre la solidaridad orientada a intentar obtener un trabajo remunerado, dada la elevada tasa actual de paro juvenil y el clima de fatalismo discursivo generado en torno a la situación laboral de las personas más jóvenes. Por otra parte, los más jóvenes de entre los jóvenes, mantienen unos vínculos sociales muy fuertes con el grupo de iguales, por ello no es de extrañar que utilice las prácticas solidarias como vehículo potenciador de sus relaciones sociales. Y no podemos dejar de añadir que se trata de una impresión que concuerda con discursos de otra naturaleza considerados en esta investigación, muy especialmente en el mundo profesional e institucional. Se corrobora con el discurso que mantienen los/as profesionales que trabajan en contacto directo con la juventud; ellos mismos han manifestado que los/as jóvenes que acuden a ellos/as muestran como principal inquietud la posible inserción al mercado laboral, sobre todo los de más edad.

Estamos pues ante una construcción social negativa afianzada y recurrente en el discurso analizado que la propia juventud andaluza hace sobre sí misma. Según los propios jóvenes, y también de acuerdo a los datos obtenidos en las fuentes secundarias, la juventud se caracteriza fundamentalmente por ser pasota, egoísta, hedonista, impulsiva y poco comprometida. Teniendo dicha premisa a la vista ¿es plausible afirmar que la juventud andaluza no se considera solidaria? Podría ser una cuestión difícil de responder sin incurrir en impresiones contrapuestas que reflejan conflictos dentro del propio discurso y las representaciones en él contenidas.

\section{Conclusiones}

Una vez desarrollado nuestro análisis de los resultados obtenidos presentamos a continuación las siguientes conclusiones.

En cuanto al término solidaridad podemos concluir que los/as jóvenes andaluces entienden que la solidaridad consiste en ayudar sin recibir nada a cambio, ponerse en el lugar del otro, compartir y hacer algo por los demás. Por lo tanto prima discursivamente la representación desinteresada y no-instrumental de la práctica solidaria. 
Debemos tener en cuenta a la hora de definir la solidaridad la variable estatus socioeconómico puesto que existe la tendencia de vincular la solidaridad con la caridad por parte de los jóvenes de estatus socioeconómico alto frente a los jóvenes con un nivel socioeconómico bajo que relacionan el término solidaridad con el término justicia.

Por su parte el/la joven andaluz tiene una representación negativa de la propia persona joven que no funciona como un estereotipo exogrupal, sino que circula y es frecuente como argumento de autorepresentación de nuestros informantes. Los mismos jóvenes andaluces se describen a sí mismos como "despreocupados", "pasotas", "egoístas", impulsivos, dormidos, etc. En este caso debemos mencionar la propensión de los/as profesionales a mantener una construcción de la juventud basada en aspectos positivos, siendo la percepción de los/as jóvenes una construcción más negativa de su propio entorno juvenil.

Respecto a las prácticas solidarias concluimos que la mayor parte de dichas prácticas que realizan los jóvenes andaluces son descritas en términos que las etiquetan como "caritativas", es decir, desprovistas de una voluntad transformadora. Las principales actividades solidarias que realiza la juventud andaluza se dirigen principalmente hacia personas mayores y niños pequeños. Además destaca la participación en actividades puntuales como donar ropa o ayudar en un banco de alimentos; siendo el cuidado de personas las prácticas solidarias en las que más participan las mujeres y las prácticas solidarias relacionadas con el medio ambiente y el deporte las más practicadas por los hombres.

Por último concluimos que las motivaciones principales por las cuales la juventud andaluza realiza prácticas solidarias son: insertarse en el mercado laboral, acudir a ciertas actividades porque van sus amigos, ayudar a otras personas que han pasado por situaciones parecidas a las suyas y vivir nuevas experiencias. Además, en menor medida, presentan las siguientes motivaciones: sentirse útiles, obtener cierta satisfacción personal, tener empatía y querer cambiar las acciones injustas.

\section{Bibliografía}

Aguinaga, J. y Comas, D. (1991). Infancia y adolescencia: la mirada de los adultos. Madrid: Ministerio de asuntos sociales.

Bergua, J.A. (1999).De-finición y de-finitación de la juventud. Una crítica de la teoría estándar. Política y sociedad, 32, 231-235.

CEPES (2010). Instantánea de la juventud andaluza 2010. Sevilla: Junta de Andalucía.

Comas, D. et al. (2003). Jóvenes y estilos de vida. Valores y riesgos en los jóvenes urbanos. Madrid: INJUVE.

Comte, A. Discurso sobre el espíritu positivo (1844). Alianza Ed. Madrid, 1980.

De Sebastián, L. (1996). La solidaridad "guardián de mi hermano. Barcelona: Editorial Ariel.

Durkheim, E. (1893). La división del trabajo social. Planeta-Agostini. Barcelona, 1985.

Fundación Santamaría. (2010). Jóvenes españoles 2010. Nota de prensa, <http://imagenes.publico-estaticos.es/resources/archivos/2010/11/24/1290602759388dossierinforme-jovenes-espanoles-2010-v3.pdf.>[18 de noviembre 2014]. 
García Roca, J. (1998). Exclusión social y contracultura de la solidaridad. Prácticas, discursos y narraciones. Madrid: Editorial HOAC.

Gil Calvo, E. (2004). La matriz del cambio: metabolismo generacional y metamorfosis de las instituciones. En Canteras, A. corrd. (2004). Los jóvenes en un mundo en transformación. Madrid: Instituto de la juventud, Ministerio de trabajo y asuntos sociales.

Gómez Serrano, P. J. (1994). ¿Jóvenes adaptados?. Revista Documentación Social, 95, 145-154.

González Anleo, J.M. (2010). Los valores de los jóvenes y su integración socio-política. En Jóvenes españoles 2010. Madrid: ediciones S.M.

Hidalgo, A. (2006). Ética, política y ciudadanía para una Europa solidaria. Eikasia, 5.

INJUVE. (2006). Juventud, solidaridad y voluntariado. Sondeo de opinión y situación de la gente joven 2006. Madrid: Ministerio de trabajo y asuntos sociales.

Montañés, M. (2000). ¿De qué hablamos cuando hablamos de juventud? Papples del FIM, 12, 99-107.

Navarro, M. y Mateo, M. J. (1994). Jóvenes y juventud. Revista Documentación Social, 95, 9-22.

Ostau de la font, F. R. (2009). La sociabilidad humana y la solidaridad como elementos culturales de la protección social. Revista diálogo de saberes. Junio - Diciembre, 25-35.

Revilla, J. C. (2001). La construcción discursiva de la juventud: lo general y lo particular. Revista Papers, 63/64, 103-122.

Rosenmayr, L. y Allerbeck, K. (1979). Youth and society. The journal of international sociological association, 2 (27), 17-36.

Sobrino, J. (2002). Solidaridad. En Conill, J. Glosario para una sociedad intercultural. Valencia: Bancaja.

Souto, S. (2007). Ser joven en la Europa de entreguerras: política, cultura y movilización. Hispania, vol. LVIII, nº. 225. DOI: http://dx.doi.org/10.3989/hispania.2007.v67.i225

Uña, O. y Hernández, A. (2004). Diccionario de sociología. Madrid: ESIC.

Vidal, M. (1996). Para aprender la solidaridad. Pamplona: Verbo Divino.

Wallace, C y Kovatcheva, S. (1998). Youth in society. The construction and deconstruction of youth in east and western Europe. Basingstoke: MacMillan. 